\title{
TRIBUNA
}

\section{La economía española: crisis y futuro'}

\section{Manuel Delgado Álvarez}

Palabras clave: España, crisis económica, variables económicas, medidas de política económica

Key words: Spain, economic crises, economic variables, policy measures

Mots clés: Espagne, crise économique, variables économiques, mesures de politique économique

\section{Introducción}

Esta conferencia lleva por título: "La economía española: crisis y futuro", y está estructurada en tres partes:

I. ANTECEDENTES: contempla, de un lado, la situación de la economía española antes de la crisis y, de otro, el impacto de la crisis internacional sobre la economía española.

II. LA SITUACIÓN ACTUAL: ¿̇cuáles han sido las consecuencias para la economía española de la crisis financiera y económica internacional?

III. EL FUTURO: retos y desafíos de la economía española.

1 Este texto reproduce la lección inaugural impartida por el autor en el acto de apertura del curso académico 2010-2011 de la Facultad de Ciencias Económicas y Empresariales - ETEA, celebrado el pasado 30 de septiembre de 2010. Este acto fue también un homenaje académico al profesor Delgado con ocasión de su jubilación al cumplir 65 años. 
Me van a permitir que dado lo limitado del tiempo en un acto como este, me centre en los principales rasgos y retos que presenta la economía en la actualidad para superar la crisis.

\section{I.Antecedentes}

\section{I.I. La situación de la economía española antes de la crisis}

La crisis financiera internacional incidió sobre la economía española cuando se estaba iniciando un proceso de ajuste tras una larga expansión impulsada por un aumento del gasto que requirió un endeudamiento creciente de familias y empresas que no era compatible con sendas realistas del coste de la financiación y del crecimiento económico. Algunos hechos lo corroboran:

1. A lo largo del periodo 1999-2006 la economía española experimentó un fuerte crecimiento -aumento del PIB del orden del 3,5\% anual-; por el contrario, en este mismo periodo se produjeron escasos avances en la productividad.

2. Durante ese periodo de expansión, la economía española acumuló una serie de desequilibrios interdependientes entre sí, que se pueden condensar en dos:

- Un abultado endeudamiento de las empresas y de las familias que, al no estar compensado suficientemente por el mayor ahorro del sector privado y del sector público, se tradujo en un elevado recurso al ahorro exterior.

- Un peso excesivo del sector inmobiliario, en términos tanto de concentración de los recursos productivos en dicho sector -muy superior a lo observado en otros países- como de la importante materialización de la riqueza de los hogares en el sector inmobiliario. (La fuerte inversión en vivienda y el prolongado e intenso crecimiento de los precios inmobiliarios provocaron que la riqueza inmobiliaria aumentara de forma significativa; sólo en 3 años, de 2002 a 2005, creció a una media anual del 18\%, en términos reales). En definitiva, el peso de la riqueza inmobiliaria sobre la riqueza total de los hogares españoles aumentó hasta el 78,7\% entre 2002 y 2005 (EEUU 43,4\%). 
3. A su vez, la expansión trajo consigo un fuerte crecimiento del empleo, que mejoró las expectativas de crecimiento de la renta disponible y, por tanto, impulsó adicionalmente la demanda de crédito. (En 2007 el empleo en el sector de la construcción representaba el 13,2\% del empleo total, 2,7 pp por encima del registrado en 1999; por otro lado, el dinamismo de la actividad vino acompañado de un rápido incremento del precio de la vivienda, que pasó de una tasa media del 1\% entre 1995 y 1997 a otro del 18\% entre 2004 y 2005).

\section{I.2 El impacto de la crisis internacional sobre la economía española}

En 2007, la economía mundial experimentó cambios importantes que quebraron el mayor periodo de crecimiento continuado desde los años sesenta. El sistema financiero internacional experimentó los momentos más difíciles en varias generaciones; las turbulencias financieras se extendieron, llegando a comprometer la solvencia de algunas entidades sistémicamente relevantes.

La escalada de la crisis financiera, en septiembre de 2008, intensificó el proceso de desapalancamiento en el que se hallaba inmerso el sistema financiero, al tiempo que agravó sus efectos sobre la actividad real, haciéndose patente una recesión económica global que alcanzó a casi todas las principales economías desarrolladas, extendiéndose a las economías emergentes y provocando que algunos países registraran tasas de variación del PIB negativas a final de 2007 y comienzos de 2008.

Las autoridades económicas reaccionaron con contundencia frente a esta conjunción de factores adversos, con el objetivo de dar soporte al sistema financiero, salvaguardar su funcionamiento y estimular la economía. Los gobiernos diseñaron planes de apoyo a los sistemas financieros y con posterioridad adoptaron paquetes de impulso fiscal relativamente ambiciosos.

Tras la intensificación y la globalización de la crisis financiera, las respuestas de política monetaria se hicieron uniformemente expansivas, instrumentándose importantes recortes en los tipos de intervención, que alcanzarían en algunos casos niveles mínimos históricos, al tiempo que las políticas de abundante provisión de liquidez y de política fiscal adquirieron un gran protagonismo.

Esta crisis mundial está empobreciendo al mundo entero; su coste es tan elevado que muchos niegan que sea real, porque es inconcebible que haya ocurrido... ¿Pensaba alguien que el todo poderoso sistema financiero norteamericano -con 
Lehman Brothers a la cabeza- podría caer de la forma que lo ha hecho o que fraudes, estafas y otros engaños -como el caso Madoff- podría darse en la tradicional, segura y moderna sociedad norteamericana?

En la economía española, las perturbaciones generadas por la grave crisis financiera y económica mundial vinieron a superponerse al proceso de ajuste interno que se había iniciado con anterioridad, y que estaba siendo impulsado, sobre todo por la corrección de los excesos del sector inmobiliario y de los altos niveles de endeudamiento del sector privado. La confluencia de ambos procesos se tradujo en: un severo aumento de la incertidumbre, un descenso vertiginoso de la confianza, un notable endurecimiento de las condiciones de acceso a la financiación, una erosión considerable de la riqueza del sector privado y un aumento muy considerable de la tasa de desempleo. Las fuerzas contractivas que desencadenaron este proceso precipitaron a la economía española en una intensa recesión, en paralelo con las tendencias recesivas globales. Este panorama se fue deteriorando por la falta de respuesta de las autoridades españolas al inicio de la misma.

\section{La situación actual}

La crisis financiera y económica internacional ha trastocado sustancialmente el escenario en el que tendrán que llevarse a cabo los ajustes que requiere la economía española.

- Durante la primera fase de la crisis financiera internacional, la desaceleración económica fue moderada pero su agudización ha puesto en marcha mecanismos de amplificación en: una percepción de pérdida de riqueza y un cambio drástico en las expectativas de familias y empresas, conformando un escenario en el que el ajuste del endeudamiento y de la exposición inmobiliaria tiene que hacerse en condiciones especialmente difíciles.

- La crisis ha modificado no sólo las expectativas de los agentes sobre las rentabilidades futuras de sus activos financieros e inmobiliarios, sino que también han afectado a sus perspectivas de rentas laborales futuras. (Así el crédito a los hogares, que estuvo creciendo a tasas superiores al $20 \%$ durante los trimestres finales de la expansión, pasó a crecer a una tasa interanual en el entorno del $5 \%$ a finales de 2008).

- Los siguientes datos expresan dicha situación: 
1. La economía española experimentó en 2009 la mayor caída de la actividad (descenso del 3,6\% del PIB) de las últimas décadas. Esta evolución estuvo en consonancia con la severidad de las tendencias contractivas globales, aunque influida también por los desequilibrios acumulados durante la fase expansiva previa. La recesión en España ha sido, pues prolongada, y el crecimiento observado en la primera parte de 2010 ha sido débil y con cierto retraso en relación con la mayoría de los países industrializados.

2. El severo deterioro de las finanzas públicas como consecuencia de la severa contracción de la actividad y de las medidas excepcionales de estímulo. (El déficit público se situó en el 11,2\% del PIB en 2009, con un aumento de más de 13 puntos porcentuales -pp- en sólo dos años). Este acusado deterioro fiscal -provocado por la contracción económica- se explica por el aumento significativo del gasto en prestaciones por desempleo, las medidas discrecionales adoptadas, la fuerte inercia del gasto público y, sobre todo, por los descensos de la recaudación ligadas al fin del auge inmobiliario que difícilmente podrá repetirse en el futuro. La deuda pública se ha situado - a finales de 2009- en el 53,2\% del PIB, frente a un mínimo del 36,2\% del PIB de dos años antes.

3. El ajuste del sector inmobiliario ha producido una intensa reducción de la actividad, una drástica pérdida de puestos de trabajo y generado un importante efecto arrastre sobre otras ramas productivas. Pese a la intensidad de la contracción el ajuste no ha concluido todavía.

4. El crédito a las empresas y a las familias ha entrado en una fase de contracción (de registro de aumentos del $20 \%$ a familias y del $30 \%$ a empresas en el momento de mayor expansión del ciclo a valores reales del $1 \%$ en el primer caso y negativos -menos 5\%-en el segundo) como resultado de un conjunto complejo de factores: la exuberancia de la expansión de las deudas; tasas de crecimiento del crédito insostenibles, elevados ratios de endeudamiento de empresas y familias (superiores a los valores medios de los países de nuestro entorno), la marcada concentración de los préstamos bancarios en el sector de la construcción, y la paralización de los mercados interbancarios ante la inexistencia de liquidez por la falta de confianza y de fiabilidad entre los propios intermediarios financieros, entre otros.

5. La crisis y la evaluación de los riesgos afectó también a los propios intermediarios financieros expuestos a su vez a unos mercados más exigentes. Las entidades de crédito españolas resistieron los primeros embates de la crisis financiera internacional desde una posición de relativa fortaleza; no 
obstante, la duración de la crisis y, sobre todo, sus efectos sobre la economía española han ido deteriorando los colchones disponibles, en un contexto en el que los niveles de apalancamiento admisibles por los mercados y los reguladores tenderán a ser menores. De ello se deriva la necesidad de acometer un proceso de redimensionamiento del sector cuya incidencia sobre la oferta de crédito tenderá a ser tanto menor cuanto más rápida y ordenada sea ésta.

6. Pero el rasgo más negativo de la economía española durante la crisis ha sido la magnitud alcanzada por el proceso de destrucción de puestos de trabajo y por el incremento del desempleo, que está recayendo principalmente sobre el empleo temporal y ha determinado un incremento muy abrupto en el número de desempleados (4,6 millones de parados en los primeros meses de 2010 , tasa de paro del $20,1 \%$ de la población activa y 2 millones de parados más desde el momento máximo de ocupación en el tercer trimestre de 2007) que ha incidido con especial gravedad sobre determinados colectivos. (El incremento del desempleo afectó de forma desigual a los distintos segmentos de la población, siendo los colectivos con mayor incremento del paro los inmigrantes y los jóvenes, en particular los que tienen menor nivel de formación, para los que la tasa de paro alcanzó el 52,2\% en los meses iniciales de 2010). Este hecho hace que el incremento de desempleo está comportando un significativo repunte de la tasa de paro de larga duración. Sin embargo, a pesar del deterioro cíclico, los salarios reales mostraron un acusado repunte, si bien su importe sobre los costes laborales por unidad de producto se vio amortiguado por el incremento de productividad, debido en gran medida, a la destrucción de empleo. (La contracción del empleo fue muy superior al retroceso de la producción en 2009, con lo que se produjo un notable incremento de la productividad aparente del trabajo, recuperación que, no obstante, tiene un componente cíclico importante, por lo que no se puede extrapolar al futuro. De otro lado, la remuneración por asalariado se incrementó en ese mismo periodo en un 4,1\% para el total de la economía, lo que representó un avance de los costes laborales reales muy sustancial y en fuerte contradicción con la situación de grave recesión económica y fuerte aumento del paro que atraviesa la economía; no obstante, el excepcional crecimiento de la productividad ayudó a moderar el comportamiento de los costes laborales por unidad de producto, que apenas crecieron en 2009: $0,7 \%$ frente a un $4,6 \%$ en 2008).

7. Existen notables carencias en investigación, desarrollo e innovación (I + D + i). Según uno de los índices mundiales más reputados de Londres (EIU), 
en su edición de 2009, sobre un ranking de 41 países, España ocupa el lugar 26.

En resumen, la existencia de importantes desequilibrios que se habían generado y acumulado en la larga fase expansiva entorpecieron el mantenimiento de un ritmo sostenido de crecimiento a medio y largo plazo, e hicieron que nuestra economía fuera particularmente sensible a los acontecimientos negativos que pudieran ocurrir; por otro lado, los excesivos niveles que han alcanzado tanto el déficit público como la tasa de paro han abierto nuevos frentes adicionales, que han complicado una economía particularmente afectada por su elevada dependencia de la financiación exterior. Por todo ello, los ajustes que tiene que realizar la economía española siguen siendo necesarios e imprescindibles. La pertenencia a la Unión Monetaria Europea ha suministrado un importante factor de protección, sin el cual todos los ajustes habrían sido más profundos y dolorosos. Por consiguiente, la intensidad del ajuste depende básicamente de: a) la capacidad para revertir las pérdidas de competitividad a través del crecimiento de la productividad, b) la moderación de los márgenes empresariales y de los costes laborales, y c) la flexibilidad del mercado de trabajo para poder absorber la reasignación sectorial del empleo. Estos ajustes son/serán difíciles y necesitan para ser eficaces un largo periodo de tiempo, eficacia que dependerá de que se produzcan avances significativos en la solución de los problemas estructurales de funcionamiento de algunos mercados (inmobiliario, laboral, ...entre otros), derivados de una regulación que no favorece suficientemente la competencia y la flexibilidad. No obstante, la recuperación de la economía española permitió que, en la década 1997-2007, se produjeran profundas transformaciones de la sociedad española, entre ellas: el gran avance hacia la internacionalización de la economía en muchos sectores o la aplicación de nuevas vías para la concertación social en algunos sectores.

\section{El futuro: retos y desafios de la economía española}

Primero el diagnóstico y luego el tratamiento: no tenemos una sola crisis sino tres: la financiera, la económica y la del paro. La crisis no terminará hasta que el desempleo decrezca, por ello hay que hacer de la creación de empleo una prioridad, pues el crecimiento, por sí solo, no creará los empleos necesarios.

La efectividad que tengan las respuestas de política económica condicionará las perspectivas de recuperación, que dependerán de la efectividad que puedan tener las medidas adoptadas por gobiernos y bancos centrales. Las acciones excepcio- 
nales que con carácter urgente se han tomado en todos los ámbitos de la política económica han estado destinadas a solventar los retos más urgentes de la crisis financiera y a evitar que el deterioro de la actividad acabe por retroalimentarse.

Más allá de afrontar de forma decidida los retos más inmediatos, resulta indispensable que se aborden en paralelo las reformas más pertinentes en el área de políticas macrofinancieras con el doble objetivo de recuperar la confianza y reconstruir unas bases más sólidas sobre las que asentar la recuperación y su sostenibilidad:

- Una regulación y una supervisión adecuada constituye la primera, y principal, línea de defensa contra la inestabilidad financiera, como ha sido especialmente subrayado en los acuerdos alcanzados por el G-20.

- Pero la experiencia reciente hace también más patente el nexo entre inestabilidad financiera y políticas macroeconómicas-particularmente la política monetaria-y refuerza el argumento de que éstas deben actuar preventivamente ante riesgos de medio y largo plazo asociados a la emergencia de desequilibrios. (De hecho, la crisis griega ha puesto de manifiesto también la necesidad de reforzar la gobernanza del área del euro y de establecer mecanismos multilaterales que aseguren una mayor coordinación de las políticas económicas nacionales).

El programa de consolidación fiscal y las medidas adoptadas para reforzar el entramado institucional de la UME son fundamentales, pero no son suficientes para devolver la economía española a una senda de alto crecimiento. Por ello, la otra pieza esencial de respuesta a la actual situación de emergencia es la adopción de una serie de reformas estructurales que impulsen la restauración de sendas de producción y del empleo sostenido. En definitiva, la recuperación de la economía española se enfrenta a importantes desafíos, que están condicionados a que se realicen los ajustes necesarios para sostener el potencial de crecimiento, lo que requiere la adopción de reformas estructurales orientadas a contener: el ajuste del empleo, incrementar la productividad y promover la orientación de las inversiones hacia sectores más productivos y con mayor potencial de crecimiento. Sólo cuando se produzcan los ajustes necesarios y se resuelvan los problemas estructurales de la economía española se podrá obtener ritmos de crecimiento sostenido acorde con el potencial existente. Entre ellos los siguientes:

- La corrección de las ineficiencias que lastran el funcionamiento del mercado laboral tiene una importancia primordial, puesto que en su configuración actual ha mostrado las dificultades que plantea para ganar competitividad y para generar empleo sostenible. Por ello se hace necesario evitar que el desempleo 
sea persistente, a través de mejoras en los sistemas de intermediación, en la formación de los desempleados y en el sistema de negociación colectiva, así como la movilidad geográfica de los desempleados. Además, debe incrementarse la calidad del capital humano y del capital tecnológico y mejorar el entorno competitivo en el que las empresas toman sus decisiones, mediante la liberalización del sector servicios y de las industrias de la red.

- El cambio de patrón productivo que se necesita para retomar tasas de crecimiento potencial elevadas exige el fomento de la inversión en sectores alternativos a la construcción, el apoyo a la innovación e investigación para el incremento de la productividad; para ello debe incrementarse el capital humano y tecnológico, aumentar la competencia y mejorar el entorno en el que las empresas desarrollan su actividad.

- Es necesario invertir más y mejor en educación y en formación para incrementar el nivel de capital humano ya que es una de las fuentes de crecimiento a largo plazo. (La evidencia empírica disponible sugiere que esta variable es la más expansiva). Lleva razón el Ministro de Educación cuando afirma Invertir en educación es caro; no hacerlo resulta carísimo.

- Para la mejora de la competencia y la liberalización sectorial, el sector servicios debe ser objeto de atención prioritaria, pues representa un porcentaje muy elevado del valor añadido y del empleo de la economía, aunque todavía inferior al observado en las economías de los países mas desarrollados. Además, engloba las actividades más dinámicas, aquellas que lideran el proceso de generación de empleo y donde tiene mejor cabida las nuevas formas de relaciones laborales.

- El saneamiento financiero del sector privado debe discurrir en paralelo con la reestructuración de las entidades de crédito, de modo que los flujos de financiación sean eficientes para apoyar los planes de gastos de empresas y familias.

- La crisis ha caído en los mismos males de las grandes reconversiones de las décadas de los ochenta y noventa: las jubilaciones anticipadas están cercenando la denominada hucha de las pensiones por lo que el sistema podría estar abocado a un desequilibrio en pocos años. Se deben evitar las cargas que pesan sobre el propio sistema: las prejubilaciones y los planes de reajuste que se hacen recaer en jubilaciones antes de la edad legalmente establecida. (Las prejubilaciones provocan un efecto perverso: cada vez existen menos afiliados activos a la SS por cada jubilado del sistema). 
Por todo ello las políticas económicas tienen un importante papel que desempeñar. A continuación se detallan un conjunto de políticas económicas que es necesario adoptar:

1. Las políticas de oferta desempeñan un papel fundamental en la salida de la crisis y en la recuperación del crecimiento a largo plazo. (El ámbito principal se encuentra en las medidas y reformas de corte estructural que mejoren la eficiencia y la rentabilidad del tejido empresarial, que faciliten la creación de empleo, aumenten la competitividad y obtengan una respuesta flexible y eficiente de la oferta).

2. Las políticas de consolidación presupuestaria y de sostenibilidad de las finanzas públicas. Un reto fundamental es la necesidad de corregir el fuerte deterioro que ha sufrido la situación presupuestaria y garantizar una rápida vuelta a una senda de estabilidad, pues, de lo contrario, la dinámica expansiva de las finanzas públicas se convertiría en un obstáculo para la recuperación. La situación actual demuestra que una buena parte del tamaño del desequilibrio fiscal es de naturaleza estructural y que debe atajarse de forma urgente con medidas de consolidación muy ambiciosas, que logren revertir la tendencia del gasto y recomponer los ingresos, por lo que se hace ineludible acometer un proceso de consolidación fiscal que frene el rápido deterioro de la deuda y evite que la carga de la deuda se incremente en mercados crecientemente sensibilizados frente a la situación de las finanzas públicas. (El Gobierno se ha comprometido a instrumentar un programa de consolidación fiscal, de acuerdo con las recomendaciones del ECOFIN-en noviembre de 2009-, cuyas directrices se plasmaron en la Actuación del Programa de Estabilidad que se presentó en enero de este año (2010); dicho Programa reconocía la existencia de un elevado déficit estructural y planteaba su reducción en el periodo 2010-2013, desde el 11,2\% del PIB en 2009 hasta situar el saldo deficitario en el $3 \%$ del PIB al final de dicho periodo, sobre la base de un pronunciado recorte del gasto público. A este objetivo responde el ambicioso paquete de medidas que aprobó el Gobierno -el 22 de mayo pasado-, que complementa los esfuerzos iniciales de consolidación incluidos en los Presupuestos Generales del Estado y en la Actualización del Programa de Estabilidad presentado en enero de este año. El esfuerzo de austeridad asumido por el Gobierno responde a la excepcionalidad de la situación y a la voluntad de enderezar la evolución de las finanzas públicas; es imprescindible, en todo caso, asegurar que los objetivos se implementen de manera estricta para mantener el nivel de credibilidad previsto y deseado). 
3. La política de reforma del mercado de trabajo necesaria para resolver cuanto antes las ineficiencias que se han puesto de manifiesto con anterioridad. Ello exige modificar algunos de los marcos institucionales vigentes y pautas de comportamiento arraigadas, (entre ellas: la excesiva segmentación del mercado determina costes elevados para la contratación estable y la estabilidad en el empleo; la movilidad geográfica se concentra-casi de manera exclusiva-en un colectivo muy amplio de trabajadores con contratos temporales, que tienen escasas posibilidades de formación y reciclaje; el sistema de negociación colectiva que, en conjunto, obstaculiza que los salarios relativos sirvan como mecanismo de señalización de la reasignación de recursos hacia los sectores con mayor potencial de desarrollo. El Gobierno ha aprobado recientemente un conjunto de medidas dirigidas a corregir las diferencias antes mencionadas; en concreto, a reducir la dualidad en la contratación y a reforzar los instrumentos de flexibilidad interna en las empresas, sin embargo, ha quedado fuera de la reforma aspectos sustanciales, en especial, el sistema de negociación colectiva).

4. La reforma del sistema de las pensiones. En una situación como la actual, y ante exigencias que la misma plantea, la adopción de reformas en el sistema de pensiones -en línea con la propuesta realizada por el Gobierno- es especialmente oportuna, porque debe permitir una mejora de sostenibilidad en el largo plazo de las finanzas públicas sin generar efectos negativos sobre el crecimiento a corto plazo. La reforma de las pensiones debe contemplar modificaciones en algunos parámetros del sistema, entre ellos: la elevación de la edad legal de jubilación, el mínimo número de años necesarios para acceder a la prestación y el periodo de cálculo de la pensión. Estas modificaciones, de llevarse a cabo, tendrían un carácter óptimo para garantizar la viabilidad del sistema.

5. La reforma del sistema financiero centrada en la reestructuración del mismo, y más concretamente del sector de las cajas de ahorros. La necesidad de redimensionamiento del sector y la convicción de que una racionalización de las estructuras institucional y productiva de muchas entidades -dada las interferencias políticas existentes- es necesaria para mantener niveles adecuados de eficiencia en un sector caracterizado por una competencia creciente, ello abonó el terreno para la creación del Fondo de Reestructuración de Ordenación Bancaria (FROB) el marco en el que muchas cajas han ido gestando acuerdos de integración, tanto a través de fusiones como de sistemas institucionales de protección (SIP). El resultado esperado es que una parte significativa de cajas de ahorros españolas haya alcanzado o esté en proceso de alcanzar 
acuerdos de integración con otras cajas (de 45 cajas existentes se reducirán a 14). Este proceso ha supuesto la mayor reordenación del sector bancario español en mucho tiempo.

6. La regulación del mercado de la vivienda, en particular, de aquellos aspectos que determinan un escaso desarrollo del segmento de alquiler, dirigidas a actuaciones que fomenten el desarrollo de este mercado y contribuyan a dar salida al "stock" de viviendas sin vender, facilitar el acceso a servicios de alojamiento y promover la movilidad regional de los trabajadores, los problemas relativos al plazo obligatorio de los contratos y el control de las rentas de alquiler.

7. En el ámbito de los servicios, los avances más significativos van dirigidos a la liberalización del sector y a la reducción de las cargas administrativas (entre otros aspectos: facilitar el libre acceso a la provisión de determinados servicios y reducir las cargas administrativas), líneas que se deberían profundizar en el Proyecto de Ley de Economía Sostenible. Además, la eliminación de trabas en el libre funcionamiento de ciertas actividades, entre ellas las de comercio al por menor. (En este sentido, la transposición de la Directiva de Servicios debe desempeñar un papel crucial en la liberalización del sector; la aprobación por el Consejo de Ministros de las leyes que inician su transposición deberían facilitar la disminución de las cargas administrativas que soportan las empresas, reduciendo sus costes y mejorando su competitividad. Dado que el tejido productivo en España se caracteriza por el reducido tamaño de sus empresas, el impacto de esta medida será superior que en el resto de la UE).

8. En el segmento de industrias de la red, los efectos de los procesos liberalizadores llevados a cabo en la última década son más visibles en el sector de las telecomunicaciones, aunque existen ciertos segmentos en los que todavía persisten algunas rigideces, que son de importancia decisiva para aprovechar las ventajas que proporciona el desarrollo de la sociedad de la información.

9. En el sector de la energía, a pesar de los numerosos cambios regulatorios que se han introducido con una finalidad liberalizadora, el grado de competencia existente de facto dista de estar a la altura de los requerimientos para que el sector pueda desplegar sus sinergias potenciales hacia el resto de la economía. Es necesario, por tanto, alcanzar mejoras efectivas en el entorno competitivo en el que se desenvuelven las empresas del sector, en línea con las 
recomendaciones de la Comisión Europea y definir el modelo energético que mejor se adecue, desde una perspectiva actual y futura, a las características del modelo productivo y de los recursos disponibles.

10. En el sector transporte se deben contemplar distintas actuaciones tendentes a incrementar la competencia en el mercado de transporte interurbano de viajeros y fomentar el transporte por ferrocarril (a través, por ejemplo, de la mejora de la conexión entre distintos medios de transporte). Estas actuaciones están contempladas en el Proyecto de Ley de Economía Sostenible.

11. La reforma del sector de las Administraciones Públicas, dada la elevada descentralización del gasto público en nuestro país, es necesario y urgente el concurso de las Administraciones Territoriales -al igual que para la Administración Central- en estos esfuerzos de austeridad; su implicación en la consecución de los objetivos presupuestarios son vitales. Por otro lado, es igualmente necesario una reforma profunda de todas las Administraciones Públicas -Central, Autonómica y Local- que reduzca de forma significativa el gasto público improductivo o al menos lo contenga y mejore la eficiencia de los servicios prestados -entre otras, las duplicidades y triplicidades de funciones existentes para prestar un mismo servicio-; esta reforma incluiría también al sector público empresarial de todas las Administraciones y la justificación de gran número de empresas que lo sustentan.

12. Finalmente, la reforma del sistema educativo y el rediseño de los incentivos para una mejor acumulación del capital tecnológico, contribuirían también a incrementar la eficiencia de los factores de producción y, por tanto, a aumentar el crecimiento potencial de la economía.

En resumen, el programa de consolidación fiscal, las medidas de reforma del mercado de trabajo, la reestructuración del sistema financiero y la propuesta de reforma del sistema de pensiones, son los ámbitos en los que se han producido avances relevantes recientemente; pero todavía queda un largo camino por recorrer y avanzar para el tan ansiado crecimiento sostenible de la economía española. Durante los meses transcurridos de 2010 ha continuado -aunque débilmente- la mejora gradual de la economía española, en un entorno caracterizado por la progresiva recuperación de la economía mundial, la continuación de los efectos de las medidas públicas y el aumento de la confianza de los agentes. 


\section{Para finalizar...}

No quisiera terminar esta Conferencia dando un panorama sombrío, de incertidumbre y de falta de expectativas sobre el futuro de la economía española, como el que nos presentan diariamente los distintos medios, analistas y otros agentes que afirman que nuestra recuperación va a ser lenta, larga y con poca vitalidad para crear empleo; si así lo hiciera faltaría a la verdad. Un hecho cierto:

De todas las crisis se ha salido, se ha resuelto más pronto o más tarde... de ésta también se saldrá.

Para contrastar este hecho cabe plantearse los siguientes interrogantes:

¿lmaginaba alguien en 1977 que 30 años después -2007- la sociedad española iba a lograr el nivel de desarrollo y bienestar alcanzados?

A esta cuestión se podría responder que los niveles logrados son debidos, en buena parte, gracias a nuestra entrada y pertenencia a la UE, lo cual es cierto, como también es cierto que nuestra permanencia en la UME ha supuesto un escudo protector para nuestra economía y que la fortaleza de la Unión Económica y Monetaria ha mejorado por los avances en la gobernanza económica; lo cual demuestra que Europa avanza, lentamente, pero avanza.

¿lmagina alguien que en las condiciones analizadas la economía española no va a superar la situación actual por la que atraviesa?

Estoy seguro que saldremos en mejores condiciones como la historia reciente nos ha mostrado, aunque el camino va a ser duro y difícil sobre todo para la creación de empleo. Para ello es necesario que se asuma que

La recuperación del potencial de desarrollo es una responsabilidad de TODOS

TODOS debemos asumir que no solo tenemos derechos sino también deberes y responsabilidades. En concreto, la recuperación de valores que hagan a la sociedad, más justa, ética y equitativa.

TODOS debemos trabajar más y mejor, esto exige esfuerzo, compromiso y eficacia. 
TODOS debemos asumir lo que somos no lo que hemos pretendido ser y no somos, ello exige austeridad, sostenibilidad y visión de futuro.

TODOS debemos ser más solidarios con los que no tienen nada o no tienen lo suficientemente para llevar una vida digna. Los Objetivos del Milenio de lucha contra la pobreza no dependen solo de los gobiernos ni de nuevos instrumentos o fórmulas enunciadas por diferentes representantes gubernamentales -una tasa a las transacciones financieras internacionales, una mayor y mejor gobernanza y respeto de los derechos humanos...- sino de la voluntad decidida de llevarlos a cabo: implementando medidas para su ejecución, seguimiento y evaluación. Los objetivos de lucha contra la pobreza y el subdesarrollo podrán conseguirse si TODOS nos comprometemos a cumplirlos, los problemas no son de fórmulas o instrumentos sino de voluntad y compromiso.

Finalmente, unas palabras respecto a la futura Universidad Loyola Andalucía. Esta futura Universidad constituye un ilusionante Proyecto, viable, posible y de una singularidad propia dentro del panorama universitario andaluz, singularidad por el carácter identitario de la Institución Promotora del Proyecto -La Compañía de Jesús- y por la apuesta por la excelencia en los ámbitos de conocimiento, investigación y pensamiento ético, para formar profesionales en diferentes áreas, cuyo objetivo último sea el conseguir un mundo mejor para TODOS.

Como todo Proyecto tiene sus riesgos e incertidumbres, sin embargo los pasos dados hasta ahora -la apuesta decidida de la Compañía de Jesús por la Universidad Loyola, la creación de la Fundación de la Universidad Loyola de Andalucía, la presentación del proyecto a las autoridades competentes y los avales recibidosauguran que la Universidad Loyola de Andalucía será una realidad más pronto que tarde.

"Toda fuerza es débil si no está unida..." de Jean de la Fontaine.

Corresponde a TODA la Comunidad de ETEA la responsabilidad de que este Proyecto se haga realidad, así como que cumpla con los objetivos perseguidos. Estoy seguro que el capital humano -personal docente, de investigación y de servicios- sabrá responder unido con entusiasmo, motivación, responsabilidad y compromiso ante este nuevo reto, al igual que lo ha hecho, siempre, en etapas anteriores.

Muchas gracias. 